implementing and evaluating public policies. It could also serve as an initial stimulus to create strong national and regional occupational monitoring systems.

\section{0-311 SETTING PRIORITIES IN OCCUPATIONAL HEALTH RESEARCH IN EUROPE}

${ }^{1}$ Manolis Kogevinas, Ingrid Sivesind Mehlum, Neil Pearce, Kurt Straif, Michelle Turner, Maria Albin, Roel Vermeulen, Robert Barouki. 'IS GLOBAL, Spain

\subsection{6/OEM-2021-EPI.103}

Background Employment is an essential component of adult life, and occupation is a major determinant of health. Despite profound changes in working life, there has been little coordinated European occupational health research.

Objectives We present results from the HERA international project funded by the EU Horizon 2020 program, to set priorities for an environment and health research agenda in the EU for 2020-2030.

Methods We contacted hundreds of researchers in Europe through an online survey. We also identified major policy needs in the health and environment/occupation nexus by contacting national, regional, and European stakeholders representing authorities, intergovernmental organizations, civil society, and the private sector through surveys and regional meetings. We applied a priori defined criteria to examine novelty, public health importance, importance to the environment, impact on policies, and potential for innovation within the sustainable development goals.

Results

Main research gaps identified include (i) Climate change and worker health; (ii) Ageing workers; (iii) New technologies and chemicals; (iv) Working time; (v) Changing employment patterns and precarious employment; (vi) Mixed exposures and biomonitoring; (vii) Work-life-balance; and (viii) Neglected occupational diseases. In addition, priority actions related to occupational health were identified such as commuting to work, tools, and infrastructure such as the development of big data, biobanks, occupational cohorts, and large population cohorts with occupational information, development of exposome type approaches, and approaches examining societal aspects on employment and productivity.

Conclusions We will discuss challenges in the identification of key areas in occupational health research that will benefit from new scientific evidence and challenges in strategies to ensure the engagement of stakeholders. This large initiative in Europe has systematically evaluated priorities through the engagement of a wide spectrum of stakeholders across the continent. A consultation process will continue over the next year to raise additional research gaps and calibrate recommendations.

\section{0-329 EFFECTIVENESS EVALUATION OF A REGULATORY TRAINING STANDARD TO PREVENT FALLS FROM HEIGHTS IN CONSTRUCTION}

'Lynda Robson, Victoria Landsman, Hyunmi Lee, Peter Smith, Benjamin C Amick, Cameron Mustard. 'Institute for Work \& Health, Canada

10.1136/OEM-2021-EPI.104
Objective The effectiveness of a regulatory training standard to prevent falls from heights in construction in the province of Ontario, Canada was evaluated. The standard specifies a full day of working-at-heights safety training, including both theoretical and practical elements, delivered by a governmentapproved training provider. Once the regulatory requirements were in full effect in fall 2017, over 400,000 workers were trained to the standard.

Methods A comprehensive effectiveness evaluation of the working-at-heights training standard was undertaken, including a longitudinal survey of over 600 workers who underwent the training, collection of their pre-post-training knowledge test scores, and a quasi-experimental analysis of lost-time injury claims for work disability insurance.

Results A large increase in mean knowledge test score (out of 10 points) was observed for workers on their day of training: from 6.8 to 9.5 points (p Follow-up after two years, showed a large decline in knowledge test scores (to mean score 7.5). In contrast, the work practice improvements had been sustained. The incidence rate of lost-time claim injuries due to falls targeted by the training (e.g. falls from roofs) declined by $21.0 \%$ from 2012-2014 to $2017-$ 2018 in the Ontario construction sector. In comparison, the rate due to falls not targeted by the training (e.g. falls at the same level) increased by $2.9 \%$; and the rate due to non-fall traumatic injuries decreased by $7.3 \%$. These differences in incidence rate changes were statistically significant. (Analyses are currently being updated to include injuries from 2019.)

Conclusion The evaluation findings provide consistent support for a conclusion that the mandatory training standard was effective in reducing the incidence of injuries targeted by the training.

\section{0-483 GEOGRAPHIC VARIATION IN WORK DISABILITY DURATION IN 5 CANADIAN WORKERS' COMPENSATION JURISDICTIONS}

${ }^{1}$ Kimberly Sharpe, Kimberlyn McGrail, Chris McLeod, Cameron Mustard. ${ }^{1}$ University of British Columbia, Canada

\subsection{6/OEM-2021-EPI.105}

Introduction Prior research has focused on individual or employer-level factors that influence work disability duration. A smaller number of studies identified differences in work disability duration by province or state and the urban-rural spectrum. Variations may also occur across smaller units of geography due to place-based factors, such as labour market characteristics and healthcare access, that play an important role in work disability duration.

Objectives The purpose of this study is to describe geographic variation in work disability duration within five work disability jurisdictions in Canada and examine if variation differs by injury type and jurisdiction.

Methods Using Canadian workers' compensation data, we examined variability in disability days by calculating the coefficient of variations (CVs) across standardized units of geography in cohorts of workers with low back, shoulder and knee injuries.

Results Preliminary results suggest shoulder injuries had the longest disability duration with a mean of 50.10 days (SD 